\title{
Efficacy of shock-absorbing versus rigid pylons for impact reduction in transtibial amputees based on laboratory, field, and outcome metrics
}

\author{
Jocelyn S. Berge, MSE; ${ }^{1}$ Joseph M. Czerniecki, MD; ${ }^{1-2}$ Glenn K. Klute, PhD ${ }^{* 1,3-4}$ \\ ${ }^{1}$ Department of Veterans Affairs (VA), Rehabilitation Research and Development Center of Excellence for Limb Loss \\ Prevention and Prosthetic Engineering, VA Puget Sound Health Care System, Seattle, WA; ${ }^{2}$ Department of Rehabilita- \\ tion Medicine, ${ }^{3}$ Department of Mechanical Engineering, and ${ }^{4}$ Department of Electrical Engineering, University of \\ Washington, Seattle, WA
}

\begin{abstract}
Prosthetic manufacturers have marketed shockabsorbing pylons (SAPs) for attenuation of injurious loads from foot-ground contact. In this study, we compared a commonly prescribed SAP with a conventional rigid pylon, using a withinsubject design ( $n=15$ unilateral transtibial amputees), to assess effect on gait mechanics, measure transmitted accelerations in situ, and determine functional outcomes using step counts and questionnaires. No differences were found across pylons for self-selected walking speed, prosthetic-side step length, prosthetic-side loading rate and decelerative peak of the vertical ground reaction force, peak pylon acceleration, step count per week, or questionnaire results that examined pylon performance and subjects' pain and fatigue levels. The only statistically significant finding was for the prosthetic-side knee angle at initial contact, where subjects displayed an average of 2.6 degrees more flexion with the rigid pylon than the SAP while walking at a controlled speed $(p=0.004)$; this result indicates that transtibial amputees are able to modulate the effective stiffness of their residual limb in response to changes in prosthetic component stiffness. The results from the laboratory, field, and subjective outcome measurements suggest that the SAP in this study is as effective as a rigid pylon for unilateral transtibial amputees.
\end{abstract}

Key words: amputation, artificial limbs, biomechanics, gait impact force, gait mechanics, prosthetics, rehabilitation, residuallimb pain, rigid pylon, shock-absorbing pylon.

\section{INTRODUCTION}

Lower-limb amputees lack many natural mechanisms that attenuate the impact forces of gait, such as a heel pad and some lower-limb joint motion. In addition, the soft tissues of the residual limb are not particularly well adapted for tolerating mechanical loading; this can lead to pain and mechanical skin injury [1]. Residual-limb discomfort is a common complaint for lower-limb amputees that affects 75 percent of transtibial amputees, with approximately 60 percent who rate their pain as moderately to severely bothersome [2].

Abbreviations: 3-D = 3-dimensional, BW = body weight, $g=$ acceleration of gravity, GRF = ground reaction force, $\mathrm{MFI}=$ Multidimensional Fatigue Inventory ${ }^{\circledR}$, SAP $=$ shock-absorbing pylon, $\mathrm{SD}=$ standard deviation, VA = Department of Veterans Affairs, VAPSHCS = VA Puget Sound Health Care System.

This material was based on work supported by the Department of Veterans Affairs, Veterans Health Administration, Rehabilitation Research and Development Service (grant A2448R).

*Address all correspondence to Glenn K. Klute, PhD; Department of Veterans Affairs, Rehabilitation Research and Development Center, 1660 South Columbian Way, MS 151, Seattle, WA 98108; 206-764-6724; fax: 206-277-3963.

Email: gklute@u.washington.edu

DOI: 10.1682/JRRD.2005.02.0034 
Prosthetic manufacturers have developed shockabsorbing pylons (SAPs) to compensate for the absence of impact-absorbing mechanisms in an amputee's lower limb. Few studies have attempted to quantify their efficacy, and most have used small sample populations of five or less [3]. Limited scientific evidence exists from which clinicians can form clinical prescription guidelines.

Two studies with sample sizes of more than five subjects have examined metabolic oxygen consumption [4] and kinematic and kinetic measures during gait [3] for quantitative performance evaluation of an SAP as compared with a conventional rigid pylon. Buckley et al. found no differences in energy expenditure (i.e., the volume of oxygen consumption) between an SAP and a conventional rigid pylon when amputees walked at self-selected speeds, but when amputees ( $n=6$ unilateral transtibial) were asked to walk much faster (160\% of self-selected speed), they expended almost 10 percent less energy [4]. Interestingly, Gard and Konz found that walking with an SAP resulted in greater gait asymmetry at self-selected speeds, but this result was not statistically significant at higher speeds ( $n=10$ unilateral transtibial) [3]. These studies suggest that for amputees who can vary their gait speed, SAPs may provide a metabolic benefit when they walk fast. However, for amputees who usually walk at one speed, the greater asymmetry exhibited with the SAP might increase secondary disabilities (e.g., low-back pain, intact-limb degenerative joint arthritis). Gard and Konz reported no statistically significant findings among the other kinematic and kinetic metrics for their grouped data as a function of walking speed, which may indicate limited efficacy of SAPs for their intended purpose, absorption of shock [3]. Surprisingly, no study has reported in situ measurements of shock attenuation (or lack thereof) for commercially available SAPs or the influence these devices might have on community and household-activity ambulation. However, encouragement for the prescription of SAP remains as studies show that subjects prefer walking with the SAP versus the conventional rigid pylon [3-4].

In this study, we compared the performance of an SAP (Mercury ${ }^{\mathrm{TM}}$ Telescopic Torsion Pylon, Chas A. Blatchford \& Sons, Ltd, Hampshire, England) with a conventional rigid pylon using a combination of laboratory, field, and subjective outcome measurements to discover why amputees seem to prefer SAPs. Specifically, our objectives were to (1) determine if an SAP affects the mechanics of amputee gait, (2) quantify the attenuation characteristics of an SAP as compared with a rigid pylon in situ, and (3) assess the functional impact of the pylons on levels of activity, pain, and fatigue among unilateral transtibial amputees.

\section{METHODS}

A myriad of parameters exist that we can use to quantify the effect an intervention or experimental condition has on gait. The challenge for gait researchers is to identify those parameters most likely to exhibit a clinically relevant response while preserving statistical power by limiting the number of parameters analyzed.

We projected that unilateral transtibial amputees would alter their gait mechanics in response to the theorized attenuation provided by the SAP. Lower-limb amputees would sense that their residual limb was experiencing smaller impact loads when walking at their self-selected speed on the SAP as compared with the rigid pylon and respond by modifying their gait. Specifically, we hypothesized that when walking on the SAP as compared with the rigid pylon, subjects would increase their self-selected walking speed and prosthetic-side step length. Since faster self-selected walking speed is correlated with increases in limb loading rates and magnitudes [5-6], we further hypothesized that the prosthetic-side loading rate and decelerative peak magnitude of vertical ground reaction force (GRF) would increase when amputees walked at their self-selected speed on the SAP as compared with the rigid pylon.

The inclusion of an SAP to the prosthetic limb would certainly increase the compliance of the limb as shown by mechanical testing and manufacturer instructions that indicate the devices should compresses approximately 5 to $8 \mathrm{~mm}$ during walking, when properly configured [7]. Analogous to the way intact subjects modulate their effective leg stiffness in response to changes in surface conditions, amputees might increase their effective leg stiffness at initial contact by decreasing knee flexion while wearing an SAP [8]. Specifically, to further assess SAP effect on gait mechanics, we hypothesized subjects would exhibit a smaller magnitude of prosthetic-side knee flexion at initial ground contact when walking at a controlled speed $( \pm 10 \%)$ of $1.2 \pm 0.12 \mathrm{~m} / \mathrm{s}$ on the SAP compared with the rigid pylon. Because we expected the selfselected speed of amputees wearing the SAP to be faster, we controlled walking speed to block effects associated with changes in gait kinematics as a function of speed [9]. 
To quantify the attenuation characteristics of an SAP in situ, we mounted an accelerometer at a proximal site on the SAP. We hypothesized that for subjects walking at $1.2 \pm$ $0.12 \mathrm{~m} / \mathrm{s}$, peak accelerations taken from an accelerometer mounted on the proximal end of the SAP would be smaller in magnitude than those from an accelerometer mounted on the rigid pylon. Again, because we expected the selfselected speed of amputees wearing the SAP to be faster, we controlled walking speed to block effects associated with changes in gait kinetics as a function of speed [5-6].

Finally, with respect to outcome measures, we hypothesized that subjects would increase their activity levels, feel more comfortable, and experience less residual-limb pain and overall fatigue while wearing the SAP compared with the rigid pylon. To measure activity levels in the community and household environment, we recorded the number of steps taken over a 1-week period. To solicit the subjective experiences of our subjects, we used questionnaire instruments to document comfort, pain, and fatigue.

\section{Patient Recruitment}

Subjects were recruited from the Department of Veterans Affairs (VA) Puget Sound Health Care System (VAPSHCS) and the Seattle metropolitan area prosthetic clinics' unilateral transtibial amputee patient populations. To be included in the study, all subjects had to be (1) between 18 and 70 years of age, (2) prosthesis users for a minimum of 2 years and at least 8 hours a day, (3) able to walk without the use of upper-limb aids, (4) at least a moderately active community ambulatory, (5) under the pylon manufacturers' published weight restriction (264 lb), and (6) free from falls within the past 6 months. Subjects were excluded if (1) the etiology of amputation was a tumor and active tumor or tumor treatment existed and/or (2) significant lower-limb pain condition or other neurological condition that would interfere with the gait pattern existed. None of the subjects wore an SAP prior to participating in the study and all provided informed consent of the protocol, which was approved by the University of Washington's Human Subjects Review Committee.

\section{Prosthetic Alignment and Fitting}

Fifteen male unilateral transtibial amputees (mean \pm standard deviation [SD] age: $51 \pm 9$ yr, body mass: $92 \pm$ $17 \mathrm{~kg}$, height: $1.79 \pm 0.06 \mathrm{~m} ; n=10$ traumatic, $n=4$ dysvascular, $n=1$ infection) were assessed by the VAPSHCS research prosthetist (a licensed, certified prosthetist and orthotist with 15 years experience). Before being tested, each subject was fitted with a lower-limb prosthesis of the following prescription: a total contact, patellar-tendon bearing socket and suspension system (their current prescription or a system that duplicated or improved upon their prescription), a rigid aluminum tube with titanium adapters, and a Seattle LightFoot ${ }^{2}$ (Seattle Systems, Poulsbo, Washington). The prosthetist then aligned the fitted prosthesis; subjects were asked to wear their own shoes during the alignment process as these would be the shoes they used during the data collection process. After proper prosthetic fit and alignment had been achieved, subjects were randomly assigned either the SAP or the rigid pylon (aluminum tube) and began an acclimation period of at least 3 weeks. The SAP was outfitted by the prosthetist with the proper torsion rod and spring, with selection based upon the subject's weight and activity level according to the manufacturer's recommendations. We then covered the study pylon with an oversized cosmetic cover to conceal the type of pylon from the subject.

\section{Data Collection}

The methods we used to measure the performance of the SAP and rigid pylon included laboratory, field, and subjective outcome metrics. The measurements collected in the laboratory included self-selected walking speed, prosthetic-side step length, prosthetic-side loading rate and decelerative peak from the vertical GRF, prostheticside knee angle at initial contact, and peak pylon acceleration. The field and outcome measurements included step count per week, an opinion of performance questionnaire, the residual-limb pain grade, and the Multidimensional Fatigue Inventory ${ }^{\complement}$ (MFI) [10-11].

Following a 3-week acclimation period, we asked each subject to come to the Motion Analysis Laboratory within the VA Rehabilitation Research and Development Center of Excellence in Limb Loss Prevention and Prosthetic Engineering at the VAPSHCS for the first gait data collection session. Upon arriving at the laboratory, the subjects doffed their prosthesis and a lightweight $(8 \mathrm{~g})$, triaxial accelerometer $\left( \pm 25 g\left[1 g=9.81 \mathrm{~m} / \mathrm{s}^{2}\right]\right.$ ) (model EGAXT3-F-25; Entran Devices, Fairfield, New Jersey) was rigidly mounted to the lateral, proximal portion of the pylon with $2 \mathrm{~mm}$ self-tapping machine screws (Figure 1). Data were collected from one accelerometer axis only, which was aligned parallel to the vertical axis of the pylon. Proximally directed accelerometer signals were positive signals. The accelerometer signals were 


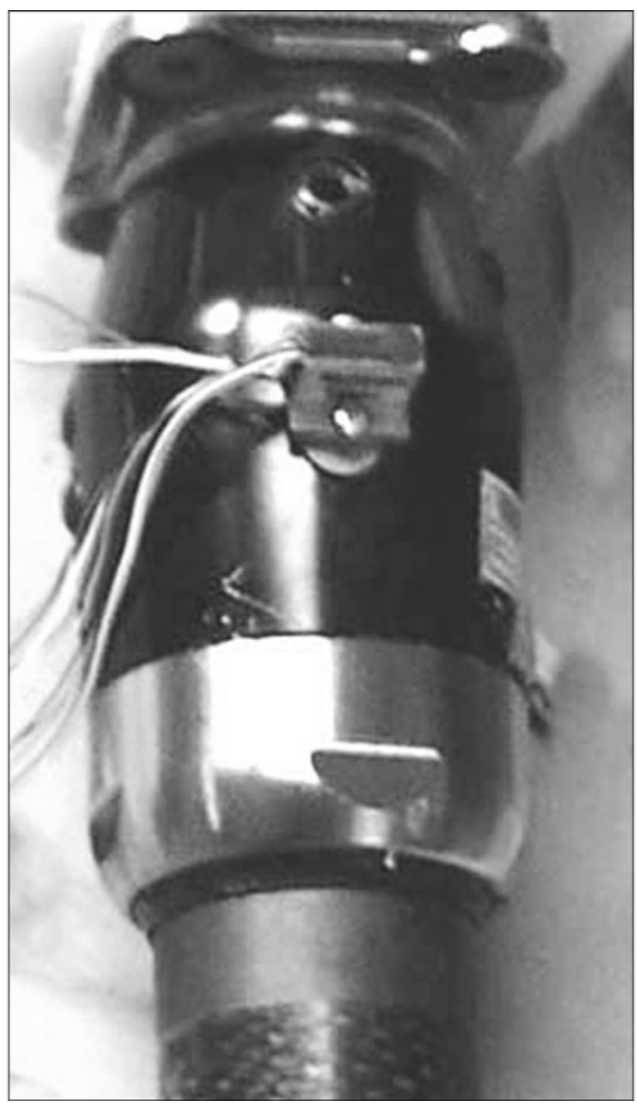

Figure 1.

Accelerometer (Entran Devices, Fairfield, New Jersey) mounted to top housing of shock-absorbing pylon. Copper washer is located under distal edge to compensate for taper of housing.

sampled at $600 \mathrm{~Hz}$ with a Vicon 612 system (Oxford Metrics, Oxford, England) that was amplified for adjustment of the working range to $\pm 5 \mathrm{~g}$ and filtered with a low-pass, two-pole Butterworth filter with a $100 \mathrm{~Hz}$ cutoff frequency (Signal Conditioning Amplifier 2310, Instruments Division, Vishay Measurements Group, Inc, Raleigh, North Carolina).

We attached reflective markers to the subject according to the 38-marker full body Vicon PlugInGait model. We placed markers on the prosthetic limb by estimating the location of missing anthropometric landmarks using the intact limb for reference. We used a configuration of 10 infrared cameras and a Vicon Workstation to sample the 3-dimensional (3-D) coordinates of each marker at $120 \mathrm{~Hz}$ while the subject walked the length of an $8 \mathrm{~m}$ walkway. Walking speed was calculated with a LabVIEW (National Instruments Corporation, Austin, Texas) virtual instrument that sampled the elapsed time between beam interruption of two infrared optical sensors (Radio Shack Corporation, Fort Worth, Texas) that were positioned $2 \mathrm{~m}$ apart along the walkway. A subject's self-selected walking speed was the average of the speeds of the three practice trials. Prosthetic-side vertical GRF data was collected with a $0.60 \times 0.90 \mathrm{~m}$ Kistler force plate (Kistler Instrumente AG, Winterthur, Switzerland). GRF data were sampled at $600 \mathrm{~Hz}$ with the Vicon 612 system, filtered with a lowpass, plug-in, two-pole Butterworth filter with a $100 \mathrm{~Hz}$ cutoff frequency (Kistler Series 5313A), and amplified with a Kistler Type 9807 amplifier.

We collected 10 acceptable trials while subjects walked first at their self-selected walking speed and then at a controlled walking speed of $1.2 \mathrm{~m} / \mathrm{s}$ (20 trials total per session). To achieve the controlled walking speed, subjects practiced with verbal feedback until at least two consecutive trials were observed within the $1.2 \pm 0.12 \mathrm{~m} / \mathrm{s}$ range with no evidence of targeting or irregular gait. A trial was accepted only if (1) walking speed was within 10 percent of the self-selected or controlled walking speeds, (2) only one prosthetic-side foot fall was noted on the force plate, and (3) no obvious signs of targeting or irregular gait were noted.

Following the laboratory data collection session, we collected the number of steps taken by subjects as they went about their daily activities over a 1-week period with a pager-sized instrument that was attached to the prosthesis at the ankle (StepWatch 2 Cyma, Seattle, Washington). We adjusted cadence and motion settings according to the subject's walking style to minimize false and/or missed counts, and the sample count recording interval was set at $1 \mathrm{~min}$.

Following the 1-week field data (step counts) collection period, subjects returned to the laboratory where three questionnaires were administered: the first questionnaire (developed in-house), for rating prosthetic performance, was termed the opinion of performance questionnaire; the second questionnaire, for evaluating residual-limb pain, was adapted from the Chronic Pain Grade [12] (residual-limb pain grade); and the third, for quantifying general fatigue, physical fatigue, and reduced activity, was the MFI [10-11]. Subjects were then fitted with the second of the two study pylons and given an acclimation period of at least 3 weeks before the aforementioned protocol was repeated with the second pylon. 


\section{Data Analysis}

Prosthetic-side knee angle (sagittal plane) and step length were calculated from kinematic data from the Vicon Workstation. During processing, we digitally filtered the kinematic data using Vicon's Woltring quintic spline filter with a mean square error of 20. The prosthetic-side step length was calculated as the distance from sound-side heel marker at heel strike to prostheticside heel marker at heel strike. The prosthetic-side knee angle at initial contact was calculated as the relative Euler angle between the femur and shank segments $\left(0^{\circ}\right.$ knee angle $=$ knee in straight-leg position, sign convention: flexion is positive and hyperextension is negative). The femur segment is defined as the axis between the hip and knee joint centers, while the shank segment is defined as the axis between the knee and ankle joint centers [13]. We used the 3-D coordinates from the external reflective markers to calculate the joint centers. This methodology precludes errors in knee angle results from vertical compression and transverse plane rotation of the SAP. The time of initial contact was extracted from the filtered angle data at the $120 \mathrm{~Hz}$ frame that was closest to prosthetic-side heel strike on the force plate.

Two variables were obtained from the prosthetic-side vertical GRF for analysis, loading rate and decelerative peak. The loading rate was calculated as the rate of force increase between $25 \mathrm{~N}$ and 0.75 times body weight (BW) [14]. The decelerative peak was defined as the peak force during single limb stance. A decelerative peak was only recorded if the amputee exhibited a typical "M" or "dualhump" vertical GRF pattern [15]. Loading rate and decelerative peak values were normalized to the subject's BW for analysis; this yielded units of BW per second and BW, respectively.

We analyzed the accelerometer data to obtain the largest magnitude acceleration (either positive or negative) during the gait cycle; we normalized this to body mass (in kilograms) to obtain acceleration per kilogram.

Following at least 1 week of field data collection, we downloaded step count data from each StepWatch 2 unit with the manufacturer-supplied docking station and software. We summed 7 continuous days of data to determine the number of steps each subject took during the week sampled.

To determine the functional impact of the test pylons, we administered three questionnaires: an opinion of performance questionnaire, the residual-limb pain grade, and the MFI. The opinion of performance questionnaire
(Figure 2) that was developed for this study allowed subjects to rate how the prosthesis performed and how they felt when they walked on that particular prosthesis. Questions were answered with an "X" marked on a $10 \mathrm{~cm}$ line, with the far left of the line indicating that a subject strongly disagreed with the question and the far right that a subject strongly agreed. The scores were calculated as measured distances (to the nearest $0.1 \mathrm{~cm}$ ) from the far left end of the line to the center of the subject's "X." Individuals' scores were tabulated for each pylon type.

We adapted the residual-limb pain grade (Figure 3) for this study from the Chronic Pain Grade [12] to specifically evaluate residual-limb pain. On a scale of 0 "No pain" to 10 "Pain as bad as could be," subjects were asked to rate their current pain intensity and their least, worst, and average pain intensity over the previous week. In addition, they were asked to rate how the pain influenced their daily activities in that week on a 10-point discrete scale of 0 "No interference/not bothersome" to 10 "Unable to carry on any activities/as bothersome as could be.” A similar chronic pain grade has been found reliable and valid in the measurement of amputee lowerlimb pain [2]. Because of our primary interest in four aspects of residual-limb pain, subjects' scores for questions regarding the intensities of their average and worst residual-limb pain (questions 5-6), residual-limb pain interference with daily activities (question 8), and how bothersome the residual-limb pain was during the

\section{I can easily do my normal day-to-day activities while wearing this prosthesis.}

Strongly Disagree Strongly Agree

2. I was very comfortable while walking on hard surfaces with this prosthesis (e.g., concrete, tile, etc.).

Strongly Disagree Strongly Agree

\section{At the end of the day, I feel like I have more energy while wearing this prosthesis.}

Strongly Disagree Strongly Agree

4. Overall, I think this prosthesis has improved my function.

\begin{tabular}{ll}
\hline Strongly Disagree & Strongly Agree
\end{tabular}

Figure 2.

Questions from opinion of performance questionnaire. 
1. In the past week, have you experienced residual-limb pain that comes and goes, constant residual-limb pain with variations in intensity, or constant residual-limb pain with little or no variation in intensity?
a. comes and goes (some pain free periods)
Please continue with question 2
b. constant pain, with variation in intensity
Please continue with question 4
c. constant pain, with little variation in intensity
Please continue with question 4

2. If your residual-limb pain is not constant, how often did you experience residual-limb pain?
a. only once or twice
b. a few times (about once/week)
c. fairly often (2-3 times/week)
d. very often (4-6 times/week)
e. every day

3. How long does your residual-limb pain usually last?
a. a few minutes
b. several minutes to an hour
c. several hours
d. a day or two
e. more than two days

4. How would you rate your residual-limb pain at the present time, that is, right now? no pain
0
2
4
5
6
7
8

pain as bad as

it could be

5. In the past week, how intense was your average residual-limb pain?

no pain
0

1

2

3

4

5

6

7

8

pain as bad as it could be

10

6. In the past week, how intense was your worst residual-limb pain? no pain

$\begin{array}{lllllllllll}0 & 1 & 2 & 3 & 4 & 5 & 6 & 7 & 8 & 9 & 10\end{array}$

7. In the past week, how intense was your least residual-limb pain?

no pain

0

12

23

4

5

6

7

8

pain as bad as it could be

10

8. In the past week, how much has your residual-limb pain interfered with your daily activities on a scale of 0 to 10 , with 0 being “no interference” and 10 being “unable to carry on any activities"?

no interference
3

4

5

6 unable to carry on any activities

10

9. In the week, on average, how bothersome was your residual-limb pain on a scale of 0 to 10 , with 0 being "not bothersome" and 10 being "as bothersome as could be"?

not bothersome

0
2

3
4

5

6

7

8

as bothersome as could be

10. About how many days in the last week have you been kept from usual activities (work, school, or housework) because of residual-limb pain? disability days.

Figure 3.

Questions from residual-limb pain grade questionnaire. 
proceeding week (question 9) were tabulated for each pylon type.

The MFI is a widely used 20-item self-report instrument that is valid for examining general, physical, and mental fatigue as well as reduced activity and reduced motivation in a variety of patient groups [10-11]. Questions were posed as statements (e.g., "I feel tired" and "I think I do a lot in a day"), and subjects respond on a 5-point discrete scale, with the far left representing, "Yes, that is true" and the far right representing, "No, that is not true." Scores for each MFI question ranged from 1 to 5 , with a higher score indicating more fatigue. A total score for each of the five subscales was calculated by summing four questions (range 4 to 20). Because we were interested in physical and general fatigue and reduced activity, we used scores on these subscales in the analysis.

We used linear mixed-effects statistical models to assess if the gait characteristics, foot-ground reaction force parameters, knee angle at initial contact, and acceleration peaks differed by pylon type, with trial number as a covariate. The comparisons at self-selected walking speed included self-selected walking speed (a dependent variable), prosthetic-side step length, and prosthetic-side loading rate and decelerative peak from vertical GRF. The comparisons at controlled walking speed included prosthetic-side knee angle at initial contact and peak pylon acceleration. The variability of these relationships across subjects was accounted for by modeling a random effect for pylon for each subject. We used paired $t$-tests to determine if step counts differed by pylon type. Differences in the responses to the opinion of performance questionnaire, the residual-limb pain grade, and the MFI by pylon type were assessed with the Wilcoxon Signed Rank Test. Significance was set before analysis at an alpha level of 0.05, and we applied the Bonferroni correction to account for multiple tests. Statistical analyses were performed with S-PLUS 6.2 (Insightful Corp, Seattle, Washington) and R 1.9.1 (Free Software Foundation, Boston, Massachusetts).

\section{RESULTS}

The self-selected walking speed mean \pm SD for this group of 15 unilateral transtibial amputees were $1.24 \pm$ $0.19 \mathrm{~m} / \mathrm{s}$ and $1.25 \pm 0.18 \mathrm{~m} / \mathrm{s}$ while walking on the rigid pylon and the SAP, respectively $(p=0.62)$. The prosthetic-side step length mean \pm SD were $0.69 \pm 0.07 \mathrm{~m}$ and $0.70 \pm 0.06 \mathrm{~m}$ for the rigid pylon and the SAP, respectively $(p=0.10)$. The mean \pm SD prosthetic-side loading rates were $8.99 \pm 2.77 \mathrm{BW} / \mathrm{s}$ and $8.99 \pm$ $2.73 \mathrm{BW} / \mathrm{s}$ for the rigid pylon and the SAP, respectively $(p=0.83)$.

The mean \pm SD decelerative peak magnitudes for the rigid pylon and SAP conditions were $1.10 \pm 0.10$ and $1.11 \pm 0.10 \mathrm{BW}$, respectively ( $p=0.83$ ). Two rigid pylon trials from one subject exhibited an atypical vertical GRF pattern, where the force rapidly achieved BW and stayed at approximately this same value throughout the stance phase. A decelerative peak could not be accurately determined for this "plateau" pattern; therefore, two missing data points were defined for the loading rate and decelerative peak statistical analysis, which caused a reduction in degrees of freedom in the statistical analysis (i.e., the linear mixed-effects model removed two of ten rigid pylon trials from one subject). Because the plateau magnitudes of these two trials were nearly the same as the decelerative peaks from this subject's other eight trials, the mean and SD calculated by including or excluding the two trials from the data set were essentially identical. Thus, the increase in the probability of performing a Type II error during the hypothesis test was negligible.

The mean \pm SD prosthetic-side knee angles at initial contact while subjects were walking at $1.2 \pm 0.12 \mathrm{~m} / \mathrm{s}$ on the rigid pylon and the SAP were $4.01^{\circ} \pm 5.97^{\circ}$ and $1.40^{\circ} \pm 5.41^{\circ}(p=0.004)$. Both means exhibit considerable variability especially compared with the other measurements. For both pylon conditions, -1 SD from the mean was a negative angle $\left(-1.96^{\circ}\right.$ and $-4.01^{\circ}$, respectively). The difference between the prosthetic-side knee angle at initial contact was the only statistically significant result from this study; subjects had, on average, $2.6^{\circ}$ less flexion with the SAP than the rigid pylon.

Measurements from the pylon-mounted accelerometer collected at the controlled walking speed revealed a large negative acceleration peak that corresponded to limb deceleration during initial contact, relatively little change from baseline during loading response through terminal stance (i.e., between $20 \%$ and $80 \%$ of stance), and a smaller, secondary peak during preswing (Figure 4). Due to several occasions of fine gauge wire breakage and a data collection hardware failure, acceleration data were available for analysis from only 10 subjects. The peak accelerations (mean $\pm \mathrm{SD}$ ) during stance were always during initial contact and were $-0.043 \pm 0.013 \mathrm{~g} / \mathrm{kg}$ and $-0.040 \pm 0.013 \mathrm{~g} / \mathrm{kg}$ 


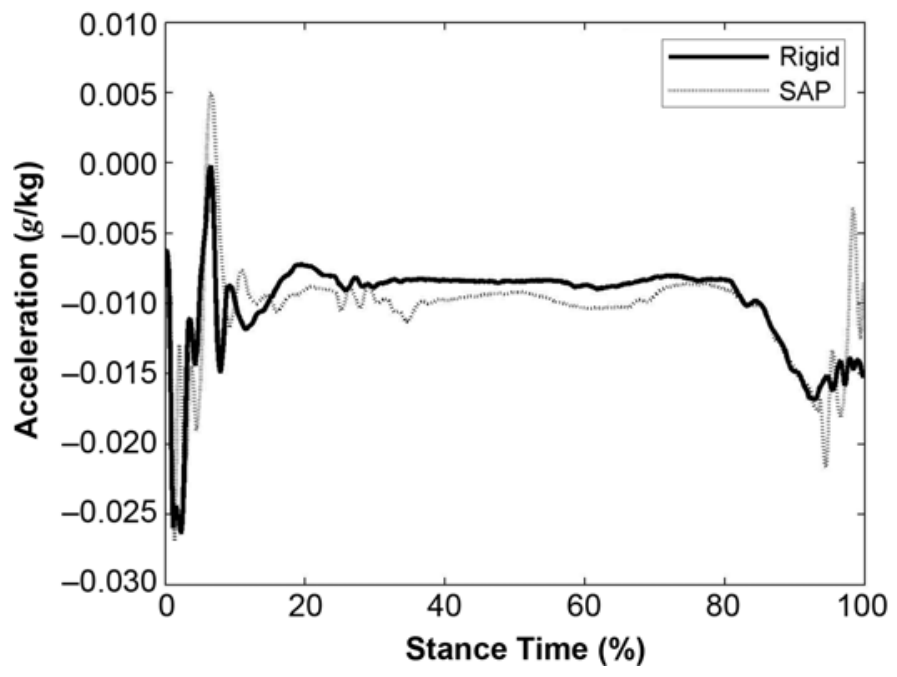

Figure 4.

Representative acceleration time series for one subject for both rigid pylon and shock-absorbing pylon (SAP). Proximally directed accelerometer signals are positive. Note large peak acceleration (negative value reveals limb deceleration) during initial contact and loading response (first $20 \%$ of stance phase).

when amputees $(n=10)$ wore the rigid pylon and the SAP, respectively ( $p=0.38)$.

The mean \pm SD number of steps taken by amputees ( $n=14$ ) during a week were 21,375 $\pm 12,810$ steps/week and 21,107 $\pm 10,995$ steps/week for the rigid pylon and the SAP, respectively ( $p=0.90)$. One subject's step count data was excluded from the analysis because of a failure of the StepWatch 2 during the week with the SAP.

The results from the kinematic, kinetic, and field measurements are summarized in Table 1. Results from the opinion of performance questionnaire analysis showed that subjects perceived no significant differences between pylons regarding ease of daily activities, comfort while walking on hard surfaces, energy level at the end of the day, and improvement of overall function (Table 2). Results regarding the intensities of subjects' average and worst residual-limb pain (questions 5-6), residual-limb pain interference with daily activities (question 8), and how bothersome the residual-limb pain was during the proceeding week (question 9) indicated no change in subjects' perceived level of pain while they were wearing the SAP as compared with the rigid pylon (Table 2). The MFI subscale responses for general fatigue, physical fatigue, and reduced activity also indicated no differences while subjects were wearing the SAP as compared with the rigid pylon (Table 2).

\section{DISCUSSION}

This study examined six gait metrics from unilateral transtibial amputees who walked with a conventional rigid pylon and an SAP. Our objective was to determine if a commonly prescribed SAP provided demonstrable effects on the mechanics of amputee gait and acceleration attenuation at the distal end of the socket as compared with a rigid pylon. In addition, we measured step counts during a week of everyday activity and administered three subjective questionnaires. For these field and outcome measurements, our objective was to assess the functional impact of pylon type on levels of activity, comfort, pain, and fatigue among unilateral transtibial amputees.

Table 1.

Laboratory and field gait measurements (mean \pm standard deviation and $p$-value) for rigid pylon and commonly prescribed shock-absorbing pylon (SAP).

\begin{tabular}{|c|c|c|c|}
\hline Gait Metric & Rigid Pylon & SAP & p-Value \\
\hline Self-Selected Walking Speed (m/s) & $1.24 \pm 0.19$ & $1.25 \pm 0.18$ & 0.62 \\
\hline Prosthetic-Side Step Length (m) ${ }^{*}$ & $0.69 \pm 0.07$ & $0.70 \pm 0.06$ & 0.10 \\
\hline Loading Rate $(\mathrm{BW} / \mathrm{s})^{*}$ & $8.99 \pm 2.77$ & $8.99 \pm 2.73$ & 0.83 \\
\hline Decelerative Peak $(\mathrm{BW})^{*}$ & $1.10 \pm 0.10$ & $1.11 \pm 0.10$ & 0.83 \\
\hline Prosthetic-Side Knee Angle at Initial Contact $\left({ }^{\circ}\right)^{\dagger}$ & $4.01 \pm 5.97$ & $1.40 \pm 5.41$ & $0.004^{\ddagger}$ \\
\hline Peak Pylon Acceleration $(\mathrm{g} / \mathrm{kg})^{\dagger}$ & $-0.043 \pm 0.013$ & $-0.040 \pm 0.013$ & 0.38 \\
\hline
\end{tabular}

${ }^{*}$ Measured at self-selected speed.

${ }^{\dagger}$ Measured at controlled walking speed $(1.2 \pm 0.12 \mathrm{~m} / \mathrm{s})$.

${ }^{\ddagger}$ Statistically significant difference.

BW = body weight. 
Table 2.

Opinion of performance (0 “Strongly Disagree” to 10 "Strongly Agree”), residual-limb pain grade (0 “No Pain/Interference” to 10 "Severe Pain/ Interference"), and Multidimensional Fatigue Inventory ${ }^{\odot}$ (4 "Less Fatigue” to 20 "More Fatigue”) questionnaire results. Question/subscale mean \pm standard deviation and $p$-value for both rigid pylon and shock-absorbing pylon (SAP).

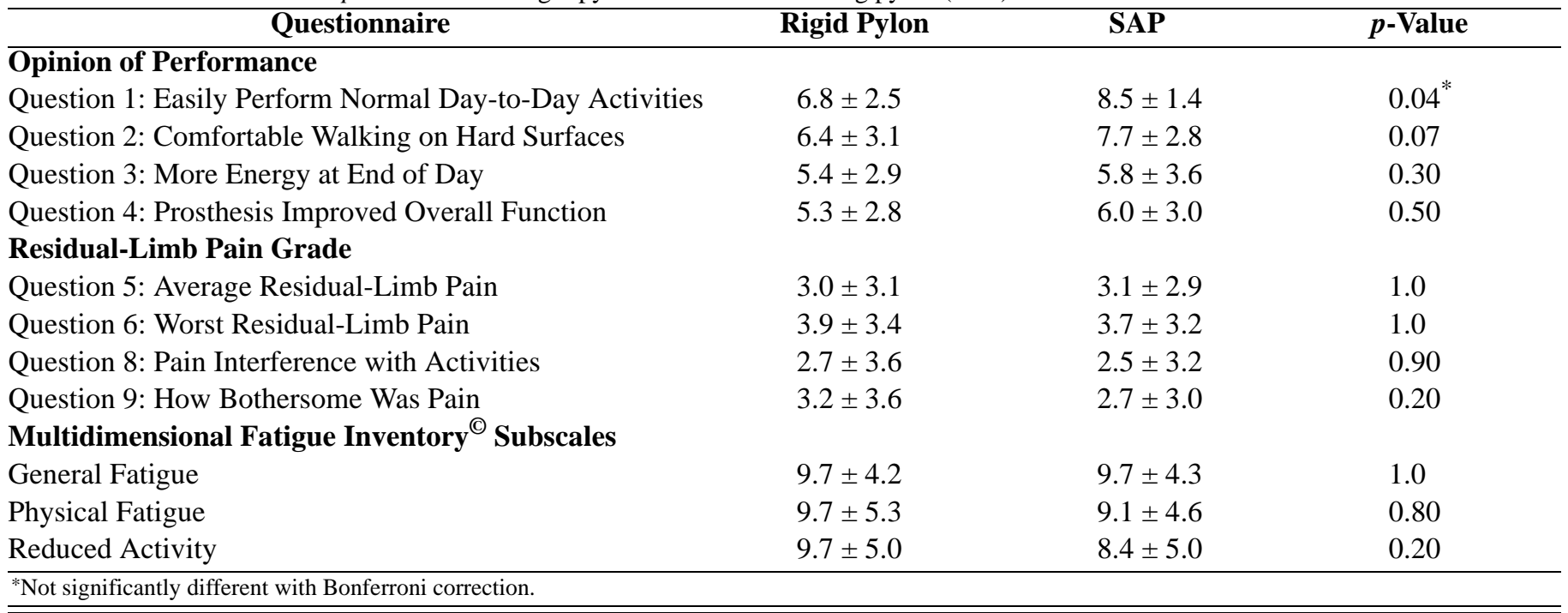

We expected that the lower-limb amputees would adapt their walking pattern in response to the theorized attenuation provided by the SAP. Subjects might sense their residual limb was experiencing smaller impact loads when walking at their self-selected speed on the SAP as compared with the rigid pylon, which would allow them to increase their speed and prosthetic-side step length. However, we observed no increase in either metric when subjects walked on the SAP compared with the rigid pylon. Gard and Konz also noted no significant difference in self-selected walking speed but did find a significant difference in a step length symmetry measure for 10 unilateral transtibial amputees who wore a rigid pylon versus an SAP (i.e., significantly more asymmetry with SAP) [3]. Gard and Konz found that their subjects walked at average self-selected speeds of 1.11 and $1.14 \mathrm{~m} / \mathrm{s}$ (no SD reported) with the rigid pylon and SAP, respectively. Mean \pm SD from our study were slightly faster but readily comparable with their study and other transtibial amputee studies [16-17] at $1.24 \pm 0.19 \mathrm{~m} / \mathrm{s}$ and $1.25 \pm 0.18 \mathrm{~m} / \mathrm{s}$ for the rigid pylon and the SAP, respectively. Unfortunately, direct comparison of our step length mean with those of Gard and Konz is not feasible, as they used a step length difference measurement (prosthetic-side step length minus sound-side step length). Their study found that when subjects walked at a selfselected speed with the SAP, they exhibited significantly more asymmetry (an average of $5.7 \mathrm{~cm}$ compared with
$3.3 \mathrm{~cm}$ ) than with the rigid pylon. However, the increase in asymmetry while subjects wore the SAP could have been because of an increase in prosthetic-side step length and/or a decrease in the sound-side step length, which may either support or refute our nonsignificant prosthetic-side step length difference result.

We detected no difference between pylons for the prosthetic-side loading rate and decelerative peak of vertical GRF while amputees walked at their self-selected walking speeds. Since self-selected walking speed is correlated with limb loading rates and magnitudes [5-6], one would expect to observe a decrease in these metrics with no change in self-selected walking speed and an effective intervention. Gard and Konz also reported no difference [3]. The mean rigid pylon loading rate of $8.5 \mathrm{BW} / \mathrm{s}$ for subjects walking at $1.24 \mathrm{~m} / \mathrm{s}$ is somewhat less than the 11.4 BW/s obtained by Coleman et al. [18]; however, their subjects were walking faster at $1.34 \mathrm{~m} / \mathrm{s}$. Likewise, the rigid pylon mean decelerative peak magnitude reported here of $1.10 \mathrm{BW}$ is in between those reported by Hermodsson et al. [19] (1.06 BW for amputees walking slower [0.85 m/s]) and Coleman et al. [18] (1.15 BW for amputees walking faster $[1.34 \mathrm{~m} / \mathrm{s}])$. The decelerative peak magnitudes reported by Gard and Konz are somewhat less than might be expected based on the reported self-selected walking speeds, $1.03 \mathrm{BW}$ at $1.11 \mathrm{~m} / \mathrm{s}$ for the rigid pylon and $1.04 \mathrm{BW}$ at $1.14 \mathrm{~m} / \mathrm{s}$ for the SAP. 
When wearing the SAP, subjects put their prostheticside knee in a more extended position at initial contact while walking at a controlled speed of $1.2 \pm 0.12 \mathrm{~m} / \mathrm{s}(p=$ 0.004). We controlled walking speed to block effects associated with gait kinematic changes as a function of speed [9]. The inclusion of an SAP to the prosthetic limb decreases limb stiffness as shown by mechanical testing and manufacturer instructions that indicate the devices should compress approximately 5 to $8 \mathrm{~mm}$ during walking when properly configured [7]. The increased knee extension at initial contact we observed while subjects walked with the SAP suggests that amputees increase their effective leg stiffness to compensate for the reduced stiffness of the shock absorber. Ferris et al. showed a related effect in running, where intact subjects modulated their leg stiffness with knee flexion and extension in response to changes in ground stiffness [20].

In comparison with intact subjects who exhibit a knee angle at initial contact of approximately $5^{\circ}[9,21]$, our results suggest that amputees exhibit a knee angle at initial contact $\left(4.01^{\circ} \pm 5.97^{\circ}\right)$ more like intact subjects when wearing a rigid pylon compared with the SAP $\left(1.40^{\circ} \pm 5.41^{\circ}\right)$. The clinical significance of this observation is unknown, but the large SD suggests caution in interpretation. One measure of treatment response, is the effect size, defined, for this type of analysis, as the difference between the mean divided by the root-mean-square of the SD [22-23]. For the prosthetic-side knee angle at initial contact, the effect size is 0.46 , which is classified by Cohen as a medium effect [24]. The mean prostheticside knee angle at initial contact reported here for the rigid pylon is somewhat less and more variable than that reported by Isakov et al. for amputees who were walking at similar speeds (mean $\pm \mathrm{SD}, 7.5^{\circ} \pm 3.6^{\circ}$ ) [17].

If the SAP provides the purported attenuation, the peak accelerations taken from the accelerometer mounted on the proximal end of the SAP would be smaller in magnitude than those from the accelerometer mounted on a rigid pylon when we controlled walking speed to block effects associated with changes in gait kinetics as a function of speed [5-6]. We observed no difference in peak accelerations across pylons at the controlled walking speed of $1.2 \pm 0.12 \mathrm{~m} / \mathrm{s}$. This unexpected result could be explained by the fact that the SAP was positioned between numerous energy dissipating systems (i.e., shoes, feet, socket liner and suspension systems, and residual-limb tissue), which all interact during locomotion. The accelerations may be well attenuated by these other systems. We found the overall mean \pm SD peak acceleration from this study $(-0.042 \pm 0.013 \mathrm{~g} / \mathrm{kg})$ comparable with the axial accelerations reported by two other studies that used accelerometers rigidly mounted to the pylons of unilateral transtibial amputees [25-26]. Van Leeuwen et al. found accelerations of 0.02 to $0.03 \mathrm{~g} / \mathrm{kg}$ (sign convention opposite to that in this study) in a study $(n=9)$ that compared two different prosthetic feet while subjects walked on a treadmill at the slower speed of $0.83 \mathrm{~m} / \mathrm{s}$ [25]. Van Jaarsveld et al. reported axial accelerations of 0.02 to $0.06 \mathrm{~g} / \mathrm{kg}$ for five amputees who walked at a self-selected speed of $1.43 \pm 0.21 \mathrm{~m} / \mathrm{s}$ with six different prosthetic feet and two different shoe types [26].

Step counts over a week that consisted of everyday activities showed no statistically significant differences when subjects wore a rigid pylon compared with an SAP. This outcome is not surprising in light of the lack of significance found with five out of six of the gait variables, but perhaps more telling is the examination of the between-subject step count distribution. Anecdotally, the amputee subjects were almost evenly divided into one of three different groups that represent three different outcomes (more steps taken with the SAP as compared with the rigid pylon, more steps taken with the rigid pylon as compared with the SAP, or the same number of steps taken). Our initial exploration of this observation found no correlated variables among those we collected; a result that suggests there may be amputees who might receive a functional benefit (or detriment) from SAP prescription. We have yet to discover the pertinent descriptors that distinguish these groups.

When we averaged the week-long sample to determine a daily step count, patients wearing the rigid pylon and the SAP walked 3,054 and 3,015 steps a day, respectively. Using componentry from the early 1980s, Holden and Fernie found that unilateral transtibial amputees ( $n=46$, average of $\sim 63$ years old) averaged 2,357 steps a day in the second year after discharge from postsurgery gait training and that age significantly affected activity level [27]. The difference in sample population age might wholly account for the difference in activity level; this suggests that modern prosthetic componentry has no influence on activity level because patients have already achieved their functional requirement. However, in a within-subject study that compared ambulatory activity across prosthetic socket liners, Coleman et al. found transtibial amputees $(n=13)$ took more steps a day while wearing a closed-cell lowdensity polyethylene liner (4,135/day) than a silicone liner 
(2,262/day) after a 3-month acclimation period [28]. The fact that Marsden and Montgomery found wide variation in activity levels between individuals but close similarities within occupations suggests a large sample population might be necessary for researchers to adequately distinguish differences between prosthetic components [29].

Responses to the opinion of performance questionnaire, residual-limb pain grade, and MFI did not show that the amputees preferred the SAP versus the rigid pylon and that the SAP did not reduce the subjects' perceived level of fatigue or pain. The outcome questionnaire results confirmed the lack of significant results found with the laboratory and field data of this study, with the sole exception being the prosthetic-side knee angle at initial contact. However, we noted in informal comments by the subjects that some had difficultly giving the study pylons a fair assessment because their opinions of each pylon were clouded by the perceived limitations of the Seattle LightFoot ${ }^{2}$. These subjects found themselves comparing the study prosthesis with their original prosthesis, which might have led to an unfair evaluation of the study pylons because most of the subjects wore higher-performing prosthetic feet as part of their original prosthesis. However, the effect of the subjects' difficulty in separating the foot from the pylon performance was perhaps negligible because the same prosthetic foot was worn for both pylon conditions.

To determine how subjects regarded the comfort provided by an SAP as compared with a rigid pylon, Gard and Konz [3] and Buckley et al. [4] both used a subjective measure in their studies of pylon preference. Gard and Konz used a 25-item questionnaire, which they developed, that contained questions regarding comfort, pain, prosthetic length and weight, pylon performance during walking and other tasks of daily living, and whether or not the subject preferred the SAP [3]. Eight of the ten subjects generally preferred the SAP versus the rigid pylon and had positive impressions regarding its effect on comfort and function in general. However, their results must be interpreted with caution as the questions may have contained a response bias toward the SAP (questions were worded to elicit positive responses for the SAP and negative responses for the rigid pylon), and no statistics were performed on the results. Buckley et al. asked six subjects to rate their comfort level, after walking at three different speeds, on a 5-point discrete scale that ranged from "Very comfortable" to "Very uncomfortable" [4]. Four subjects preferred the SAP, while the other two could perceive no difference. However, subjects were provided with a limited acclimation period of less than 10 minutes, which may have been insufficient for them to accurately perceive a difference.

We used known methods and instruments to measure the efficacy of the SAP; however, several limitations exist that should be considered when the results are interpreted. In this study, we did not control for shoe type across subjects, because the participation burden would have been too great over the length of the entire study. The choice of SAP for this study introduced a confounding factor because of its rotation capacity $\left( \pm 30^{\circ}\right.$ of internal and external rotation). This may have affected the results, but we felt it was important to study the SAP in its prescribed condition rather than alter it to eliminate rotation. The torsional characteristics of the SAP from mechanical testing have been reported [30], and future work will explore the effect of rotational stiffness on amputee gait. The step count results were subject to lifestyle (e.g., busy or slow week) and seasonal variations. However, because the study pylons were assigned in random order, these effects were minimized as much as possible. The opinion of performance questionnaire we used to quantify subjects' impressions of the prosthetic performance is not a validated questionnaire, and the questionnaire contains all positively worded questions, which may result in a scale bias. However, the same questionnaire was used for both pylons, therefore a response bias for a particular pylon was avoided. The residual-limb pain grade suffered from a "floor effect," in that subjects with no pain could not experience a reduction in pain as a direct result of the study pylon.

\section{CONCLUSIONS}

Prosthesis manufacturers have designed and marketed SAPs that purportedly absorb potentially harmful impact loads generated during walking. To explore the efficacy of these devices, we conducted a within-subject experiment to compare the performance of a commonly prescribed SAP with a conventional rigid pylon using a combination of laboratory, field, and subjective outcome measurements. Our objectives were to measure any differences in the mechanics of amputee gait, quantify the transmitted accelerations in situ, and assess the functional outcomes across levels of activity, pain, and fatigue among unilateral transtibial amputees. 
The self-selected walking speed, prosthetic-side step length, prosthetic-side loading rate and decelerative peak of the vertical GRF, peak pylon acceleration, step count per week, and subjective outcome measures of comfort, pain, and fatigue were similar for both pylons with no statistically significant differences for any of these measures. The only statistically significant result $(p=0.004)$ was the difference between prosthetic-side knee angles at initial contact; an average of $2.6^{\circ}$ more flexion was noted with the rigid pylon compared with the SAP while subjects walked at the controlled speed of $1.2 \pm 0.12 \mathrm{~m} / \mathrm{s}$. This result suggests that transtibial amputees are able to modulate the effective stiffness of their residual limb in response to changes in prosthetic limb stiffness.

These results from the laboratory measurements of gait mechanics and transmitted accelerations, the field measurements of activity, and the subjective outcome questionnaires suggest that the SAP we used in this study is as effective as a rigid pylon for unilateral transtibial amputees.

\section{ACKNOWLEDGMENTS}

We would like to thank Michael Orendurff, Janice Pecoraro, and Ava Segal for their assistance with data collection and data reduction; Jane Shofer for performing the statistical analysis; and Martin McDowell for fitting and aligning the prosthetic limbs used in this study.

\section{REFERENCES}

1. Goldstein B, Sanders J. Skin response to repetitive mechanical stress: A new experimental model in pig. Arch Phys Med Rehabil. 1998;79(3):265-72.

2. Ehde DM, Czerniecki JM, Smith DG, Campbell KM, Edwards WT, Jensen MP, Robinson LR. Chronic phantom sensations, phantom pain, residual limb pain, and other regional pain after lower limb amputation. Arch Phys Med Rehabil. 2000;81(8):1039-44.

3. Gard SA, Konz RJ. The effect of a shock-absorbing pylon on the gait of persons with unilateral transtibial amputation. J Rehabil Res Dev. 2003;40(2):109-24.

4. Buckley JG, Jones SF, Birch KM. Oxygen consumption during ambulation: Comparison of using a prosthesis fitted with and without a tele-torsion device. Arch Phys Med Rehabil. 2002;83(4):576-80.

5. Collins JJ, Whittle MW. Influence of gait parameters on the loading of the lower limb. J Biomed Eng. 1989;11(5):409-12.
6. Nilsson J, Thorstensson A. Ground reaction forces at different speeds of human walking and running. Acta Physiol Scand. 1989;136(2):217-27.

7. Berge JS, Klute GK, Czerniecki JM. Mechanical properties of shock-absorbing pylons used in transtibial prostheses. J Biomech Eng. 2004;126(1):120-22.

8. Farley CT, Houdijk HH, Van Strien C, Louie M. Mechanism of leg stiffness adjustment for hopping on surfaces of different stiffnesses. J Appl Physiol. 1998;85(3):1044-55.

9. Murray MP, Kory RC, Clarkson BH, Sepic SB. Comparison of free and fast speed walking patterns of normal men. Am J Phys Med. 1966;45(1):8-23.

10. Smets EM, Garssen B, Bonke B, De Haes JC. The Multidimensional Fatigue Inventory (MFI) psychometric qualities of an instrument to assess fatigue. J Psychosom Res. 1995;39(3):315-25.

11. Smets EM, Garssen B, Cull A, De Haes JC. Application of the Multidimensional Fatigue Inventory (MFI-20) in cancer patients receiving radiotherapy. Br J Cancer. 1996; 73(2):241-45.

12. Von Korff M, Ormel J, Keefe FJ, Dworkin SF. Grading the severity of chronic pain. Pain. 1992;50(2):133-49.

13. ViconPeak. PlugInGait Model manual, 2002. Oxford (England): ViconPeak; 2002.

14. McCaw ST, Heil ME, Hamill J. The effect of comments about shoe construction on impact forces during walking. Med Sci Sports Exerc. 2000;32(7):1258-64.

15. Schneider E, Chao EY. Fourier analysis of ground reaction forces in normals and patients with knee joint disease. J Biomech. 1983;16(8):591-601.

16. Robinson JL, Smidt GL, Arora JS. Accelerographic, temporal, and distance gait factors in below-knee amputees. Phys Ther. 1977;57(8):898-904.

17. Isakov E, Keren O, Benjuya N. Trans-tibial amputee gait: Time-distance parameters and EMG activity. Prosthet Orthot Int. 2000;24(3):216-20.

18. Coleman KL, Boone DA, Smith DG, Czerniecki JM. Effect of trans-tibial prosthesis pylon flexibility on ground reaction forces during gait. Prosthet Orthot Int. 2001;25(3): 195-201.

19. Hermodsson Y, Ekdahl C, Persson BM, Roxendal G. Gait in male trans-tibial amputees: A comparative study with healthy subjects in relation to walking speed. Prosthet Orthot Int. 1994;18(2):68-77.

20. Ferris DP, Louie M, Farley CT. Running in the real world: Adjusting leg stiffness for different surfaces. Proc Biol Sci. 1998;265(1400):989-94.

21. Kadaba MP, Ramakrishnan HK, Wootten ME, Gainey J, Gorton G, Cochran GV. Repeatability of kinematic, kinetic, and electromyographic data in normal adult gait. J Orthop Res. 1989;7(6):849-60.

22. Dunlap WP, Cortina JM, Vaslow JB, Burke MJ. Metaanalysis of experiments with matched groups or repeated measures designs. Psychol Methods. 1996;1(2):170-77. 
23. Rosnow RL, Rosenthal R. Computing contrasts, effect sizes, and counternulls on other people's published data. Psychol Methods. 1996;1(4):331-40.

24. Cohen J. Statistical power analysis for the behavior sciences. 2nd ed. Hillsdale (NJ): Lawrence Earlbaum Associates; 1988.

25. Van Leeuwen JL, Speth LA, Daanen HA. Shock absorption of below-knee prostheses: A comparison between the SACH and the Multiflex foot. J Biomech. 1990;23(5):441-46.

26. Van Jaarsveld HW, Grootenboer HJ, De Vries J. Accelerations due to impact at heel strike using below-knee prosthesis. Prosthet Orthot Int. 1990;14(2):63-66.

27. Holden JM, Fernie GR. Extent of artificial limb use following rehabilitation. J Orthop Res. 1987;5(4):562-68.

28. Coleman KL, Boone D, Smith DG, Laing L, Mathews D, Czerniecki JM. Cross-over trial comparing Alpha liner with pelite liner for trans-tibial prostheses using ambula- tory activity and questionnaire responses [abstract]. Proceedings of the 10th World Congress of the International Society for Prosthetics and Orthotics; 2001 Jul 1-6; Glasgow, Scotland. Copenhagen (Denmark): International Society for Prosthetics and Orthotics; 2001. p. W05-5.

29. Marsden JP, Montgomery SR. A general survey of the walking habits of individuals. Ergonomics. 1972;15(4):439-51.

30. Flick KC, Orendurff MS, Berge JS, Segal AD, Klute GK. Comparison of human turning gait with the mechanical performance of lower limb prosthetic transverse rotation adaptors. Prosthet Orthot Int. 2005;(1):73-81. Erratum in: Prosthet Orthot Int 2005;(2):202.

Submitted for publication February 2, 2005. Accepted in revised form August 1, 2005. 